\title{
The authentication and repatriation of a ceremonial tsantsa to its country of origin (Ecuador)
}

\author{
Craig D. Byron ${ }^{1 *+}$ (D), Adam M. Kiefer ${ }^{2+}$, Joanna Thomas ${ }^{3}$, Sagar Patel ${ }^{3}$, Amy Jenkins $^{2}$, Anthony L. Fratino ${ }^{4}$ and \\ Todd Anderson ${ }^{5}$
}

\begin{abstract}
Tsantsas, commonly referred to as "shrunken heads", are unique and valuable antiquities that were produced by the Shuar, Achuar, Awajún/Aguaruna, Wampís/Huambisa, and Candoshi-Shampra (SAAWC) peoples until the mid twentieth century. Originally used with ceremonial purpose during important social group functions, tsantsas became monetarily valuable as keepsakes and curios during the nineteenth century as a result of Western/European cultural encroachment. Unmet demand resulted in the production of convincing forgeries that created confusion about the authenticity of existing collections. Ceremonial tsantsas are both human remains and valuable cultural items. Their production and purpose were negatively influenced by colonialism and the outside curio market; as such many institutions may choose to repatriate them to their places of origin. Herein, we report on the authentication and repatriation of a ceremonial tsantsa recently rediscovered in a university's natural history collection. The personal memoirs of the original collector describe that it originated in the Ecuadorean Amazon. In consultation with the government of Ecuador, it was determined that authentication was required prior to repatriation. The tsantsa was compared to a list of thirty-three distinct characteristics shared by ceremonial tsantsas. These criteria were taken from those provided by Ecuador's National Cultural Heritage Institute and those found in prior studies from the academic literature; they include notable skin and hair features, structure and facial anatomy, and evidence of traditional fabrication and modification. We were able to affirm 30 of the 33 authenticating indicators. As part of our protocol the tsantsa was scanned with a CT-imaging system. The resulting 3D images were invaluable in discerning subtle and difficult-to-visualize characteristics regarding the skin and patterns of modification, although there are limitations related to the small size of filamentous structures. The results of the authentication were accepted by the Ecuadorean government, and the tsantsa was repatriated in June 2019. An additional benefit of CT-imaging is that data files can be generated in order to create rapid prototypes via 3D printing. In fact, as our work demonstrates, artifacts of value can be repatriated while simultaneously preserved as a volumetric replica for the purposes of a collection.
\end{abstract}

Keywords: Tsantsa, Jivaro, SAAWC peoples, Ecuador, Repatriation, Computed tomography, 3D printing

\footnotetext{
${ }^{*}$ Correspondence: byron_cd@mercer.edu

${ }^{\dagger}$ Craig D. Byron and Adam M. Kiefer contributed equally to this work

1 Department of Biology, College of Liberal Arts and Sciences, Mercer University, Macon, GA, USA

Full list of author information is available at the end of the article
}

\begin{abstract}
Introduction
Tsantsas, commonly referred to as shrunken heads, are rare cultural artifacts that were made from human remains by certain indigenous culture groups of Ecuador and Peru. Typically, male members of the Amazonian Shuar, Achuar, Awajún/Aguaruna, Wampís/Huambisa, and Candoshi-Shampra (collectively referred to as the SAAWC culture groups)
\end{abstract}

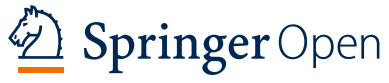

(c) The Author(s) 2021, corrected publication 2021. This article is licensed under a Creative Commons Attribution 4.0 International License which permits use, sharing, adaptation, distribution and reproduction in any medium or format, as long as you give appropriate credit to the original author(s) and the source, provide a link to the Creative Commons licence, and indicate if changes were made. The images or other third party material in this article are included in the article's Creative Commons licence, unless indicated otherwise in a credit line to the material. If material is not included in the article's Creative Commons licence and your intended use is not permitted by statutory regulation or exceeds the permitted use, you will need to obtain permission directly from the copyright holder. To view a copy of this licence, visit http://creativecommons.org/licenses/by/4.0/. The Creative Commons Public Domain Dedication waiver (http://creativeco mmons.org/publicdomain/zero/1.0/) applies to the data made available in this article, unless otherwise stated in a credit line to the data. 
manufactured tsantsas from the cranial integument (i.e., skin) of enemies slain during combat [1-3]. It was believed that tsantsas contained the spirit of the victim and all their technical knowledge and thus were considered to possess supernatural qualities and represent a source of personal power for the owner [4-6]. This power could be transferred to a host's household during ceremonies that involved a feast with many dozens of attendees. While these "ceremonial" tsantsas were of value to the Shuar before the ceremony, upon completion of the ceremony they were of little value other than as a keepsake. Tsantsas are viewed as having played an important role in facilitating social exchange across the Amazonian regions of Ecuador and Peru [7].

Ceremonial tsantsas were generated by a labor-intensive, multi-step process typically taught intergenerationally from father to son [8]. The traditional process is well described in the literature, although some variation is noted between sources. It began when the head of a deceased adversary was removed as close to the shoulders as possible, the hair was parted on the back of the skull, and an incision was made from the top of the head to the back of the base of the neck. The integument at the base of the neck was pulled back and carefully separated from the cranium, removing muscle and hypodermal connective tissues from the dermal surface. The outer two integumentary layers were subsequently inverted and the eyelids sewn shut internally using natural fibers. After the epidermis was reverted to the exterior the head was placed in cool water, then simmered in water to remove adventitious fat and grease, then once again inverted and scraped. Again, the epidermis was reverted to the exterior of the tsantsa, and the back of the head sewn together using vegetal fibers. The head was then filled through the neck opening with hot stones or sand, and hot stones are used to iron the cheeks. The head was manipulated by hand during the shrinking process so that the hot sand and stones are dispersed, ensuring even contraction of skin tissues. The tsantsa was then smoked over a fire [8-12].

During the nineteenth century, Euro-colonial valuation of tsantsas as a curio-style keepsake drove a new economy where these artifacts had significant monetary value. Tsantsa owners were willing to trade their post-ceremony tsantsas after their power had already been transferred. Because ceremonial tsantsas were produced by a distinct cultural group, authentic artifacts share a common suite of attributes associated with traditional production methods. However, the demand for tsantsas soon outstripped the supply and a marketplace for forgeries was opened; the resulting inauthentic, "commercial" tsantsas rarely share the characteristics of authentic ceremonial tsantsas in full. Commercial tsantsas were produced not for intrinsic social/supernatural power, but for export to the Euro-colonial market [2, 13, 14].

Because of their trade value, Mutter reported that ceremonial tsantsas were occasionally made from the heads of European victims in order to produce a commercial version explicitly used for trade. Additionally, taxidermists, physicians, and morticians were often known to craft versions that were even further removed from authentic ceremonial manufacture (i.e., forgeries). Heads could also be constructed from animals (sloths, monkeys, etc.) $[12,15]$, and later from synthetic materials. Regardless of authenticity, shrunken heads were exported throughout Europe and North America. By the mid-twentieth century, the colonization of Amazonian culture groups by their incorporation into larger regional states, mostly ended all tsantsa production associated with human remains, representing a significant shift in the social dynamics of the Shuar people [16].

During a recent transition into a new science facility on the campus of Mercer University, faculty found numerous avian and mammalian natural history specimens collected during the middle of the twentieth century. These specimens were organized and transferred to other institutions with similar vertebrate zoology collections [17]. During the cataloging process, one specimen was suspected to be tissue of human origin and was preliminarily identified as a tsantsa. The Mercer tsantsa was recorded as being a ceremonial tsantsa collected in Ecuador in 1942 by a now-deceased faculty member [18]. Ceremonial tsantsas are not only rare cultural artifacts produced with endemic materials and methods, but are also human remains that must be handled with respect and with an appreciation of their humanity, antiquity, rarity, and fragility. As such we sought to 1) authenticate this artifact and 2) locate an appropriate collection where it could be curated $[4,19]$. In the spirit of the Native American Graves Protection and Repatriation Act, the US legislative guidelines for appropriate handling of indigenous cultural remains, repatriation to its country of origin was of particular importance to the University [20]. After a phone conference with the Ecuadorian Embassy, the Ministry of Cultural Affairs, and the National Cultural Heritage Institute, it was decided that the University would conduct a preliminary authentication of the artifact. Upon receipt of a report summarizing the findings, Ecuador's National Cultural Heritage Institute (Instituto Nacional de Patrimonio Cultural, INPC) would determine whether or not to repatriate the tsantsa to Ecuador.

\section{Methods/experimental}

The singular artifact in this paper is presumed to be an authentic tsantsa composed of human tissue. Being of a historical nature, we are not able to identify this tissue 
and /or seek consent. Mercer University Institutional Review Board (IRB) determined that this study was exempt from IRB approval (H2007176).

The known history of the Mercer tsantsa begins in 1942, when it was procured by a now-deceased Mercer faculty member during travels in Ecuador while serving in the United States military. In his memoirs he describes an interaction he had with men of a group from the Chicham linguistic family (he described them as "Jivaros") during which he traded for the tsantsa with some of his own possessions including coins, a pocket knife, and a military insignia [18]. Following this encounter, the interior cavity of the tsantsa was filled with Ecuadorian newspaper and brought to the United States. The tsantsa was displayed amongst other items of cultural and natural history significance in the Willet Science Center at Mercer University before ultimately placing it in the University's small cultural museum. In 1979 a John Huston movie was filmed in Macon, GA close to the campus of Mercer University. The Mercer tsantsa was loaned to the motion picture production during filming [21, 22]. During several scenes, the tsantsa is clearly visible and was handled by various actors. It was returned to the museum where it remained for years before it was taken out of display and placed in storage. The exterior and interior surfaces of the tsantsa were inspected, and samples were collected of the hair and dermis. Hair samples were mounted as histological slides from which ten hair shaft and hair medulla diameters were measured.

A checklist was generated that included criteria for assessment from Ecuador's INPC. These criteria are required to be assessed for authentic tsantsas to be repatriated. Additional criteria, described in the primary academic literature, were also added to the checklist, bringing the total number of criteria to thirty-three. While there are numerous papers in the primary literature highlighting the standards for identifying ceremonial tsantsas, we selected identifying criteria from two recently published papers that focused on features and authentication of ceremonial tsantsas [2, 12]. These papers built upon other prior work by using observable characteristics to help establish the authenticity of a tsantsa, as well as present new critical observations. Charlier and coworkers [12] published the first complete procedure for identifying an authentic ceremonial tsantsa, consisting of 14 criteria derived from an analysis of 20 authentic tsantsas. In 2016 Houlton and Wilkinson [2] published additional characteristics for identifying authentic ceremonial tsantsas. For our work, we further organized an evaluation checklist. The 33 criteria compiled from these papers were organized into four categories: skin; structure and facial anatomy; evidence of traditional fabrication and modification; and hair. Some criteria could be placed into more than one category. For example, Charlier and coworkers [12] identify that authentic tsantsas possess an "oval shape of the neck in cross-section and/or lateral compression of the head." As written, the two observations in this criterion involve both structure and modification during processing, and therefore could not be separated. When this occurred, the criterion was only assigned to one category. Observations of the tsantsa in question were recorded and compared to the checklist (Table 1).

On February 20, 2019 the tsantsa was transported to Baptist MD Anderson Cancer Center in Jacksonville, FL for imaging with computed tomography (CT). A professional radiographer operated a GE Healthcare Lightspeed 16 slice CT scanner at 100 kilovolts $(\mathrm{kV}), 175$ milliamperes $(\mathrm{mA})$, a slice thickness of 625 micrometers $(\mu \mathrm{m})$, and a scan field of view that was $220 \mathrm{~mm}$. These parameters yield a voxel size of $115 \mu \mathrm{m}$. The DICOM (Digital Imaging and Communications in Medicine) files obtained from the $\mathrm{CT}$ were preliminarily reviewed in Florida following the scan on a GE Healthcare Advanced Workstation utilizing Volume Viewer to render a 3D model of the tsantsa. All subsequent 3D volume and 3D surface renderings of the DICOM files were generated and processed with OsiriX Lite. Two-dimensional sagittal cross section images of the CT scan were generated in OsiriX Lite in Maximum Intensity Projection (MIP) thick slab mode with section thickness selected between $0.4 \mathrm{~mm}$ to $25 \mathrm{~mm}$. The 3D volume rendering was generated with VR-Endoscopy settings $(\mathrm{WL}=-578, \mathrm{WW}=779)$ and any unwanted objects in the rendering, such as the mount the head was placed on during the scan, were removed using the scissor function. The removal of the extraneous objects in the 3D volume rendering carried over to the 3D surface rendering. This allowed manipulation of the pixel cutoff to visualize the tsantsa with hair (pixel $=-935$ ) or without hair (pixel $=-525$ ). Other pertinent settings in the 3D surface rendering were resolution (high), decimate-resolution (0.3), and smoothiterations (10). With the permission of Ecuador's INPC, the scan in support of the stl file is stored at the MorphoSource data repository and can be made available for download for scholarly purposes (Media 000166699; https://www.morphosource.org).

After data and image processing, a copy of the tsantsa 3D surface rendering without hair was opened in Meshmixer software directly from OsiriX Lite. Automated and manual repairs of non-manifolds and mesh gaps were performed in Meshmixer prior to export as a stereolithography file (.stl) for 3D printing (Additional file 1: File S1). The hairless tsantsa .stl was sliced, oriented and supported in Formlabs PreForm Software according to standard settings for printing with Formlabs White resin. 


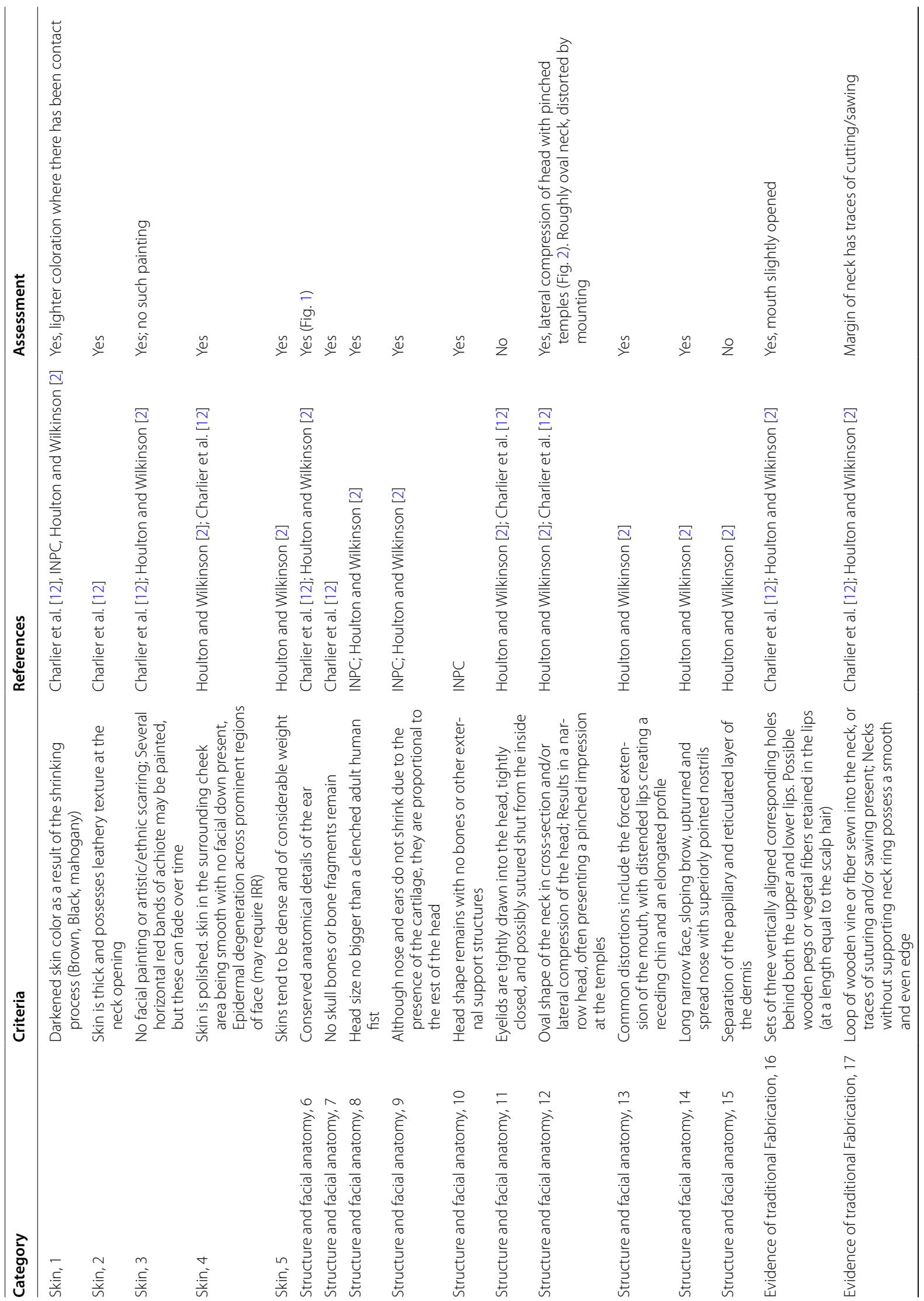




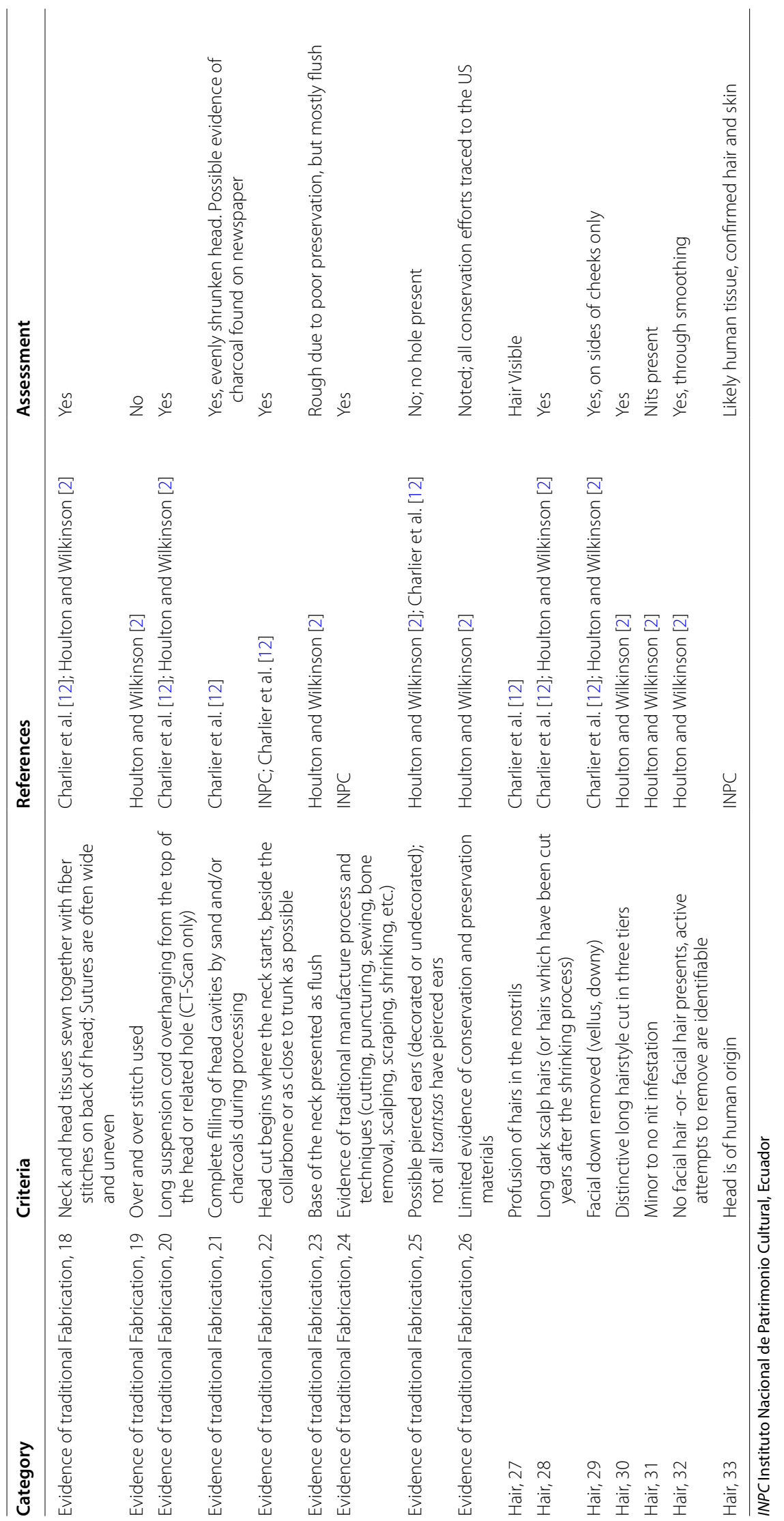


The tsantsa PreForm file was then uploaded to a Formlabs Form2, a stereolithography (SLA) 3D printer, and printed with Formlabs White resin at a layer height of $100 \mu \mathrm{m}$.

\section{Results and discussion}

Repatriation of the Mercer tsantsa to Ecuador required the generation of a report that 1) provided evidence of the provenance of the artifact and 2) compared the artifact to ceremonial tsantsas using criteria provided by the INPC. From this report, the INPC would ultimately decide whether or not to repatriate the tsantsa. To assist in authenticating the artifact, additional criteria were taken from the literature. The authenticity of tsantsas have previously been confirmed by qualitative assessment $[10,12$, 23 ], morphometric analysis [24], and genetic testing [15, $25,26]$. These papers define ceremonial tsantsas as those produced by the SAAWC culture groups using traditional methods, while commercial tsantsas are a broadly defined category of artifacts originating in the nineteenth and twentieth centuries and produced for profit as opposed to meaningful cultural purposes. Commercial tsantsas may be constructed from a human corpse, an animal corpse, synthetic materials, or some combination thereof. The preparation of the commercial tsantsa does not follow traditional methods, but may be a convincing forgery. As a result of high-quality commercial tsantsas produced from human remains, some researchers have generated a third category of tsantsa referred to as "ambiguous" [2, 3, 24]. The term "ambiguous" does not designate a tsantsa as being commercial; rather, ambiguous tsantsas possess some, but not all, characteristics of ceremonial tsantsas with known origins.

\section{Initial inspection of the Mercer Tsantsa}

The examination of the tsantsa began with an inspection of its container and separation of the head from its mount (Fig. 1a). Prior to 1979, the tsantsa was stored in an airtight glass bell jar; the original container was discarded during the filming of the movie, and at some time in 1979 the tsantsa was mounted on a base made from a painted Styrofoam cup and stored in a plastic bell jar [22]. The neck was directly attached to the mount via an unknown adhesive. After the tsantsa was separated from the mount, remnants of the mount were gently separated from the neck. The container smelled of naphthalene, but no source was noted. The cavity of the head was filled with Ecuadorian newspaper from the 1940s. The newspaper was carefully removed with tweezers, and a fine black powder was found on the paper (Additional file 2: Figure S2). Charlier and coworkers [12] note that traditional preparation of a tsantsa is known to leave small amounts of charcoal or sand in the cavity. While this fine

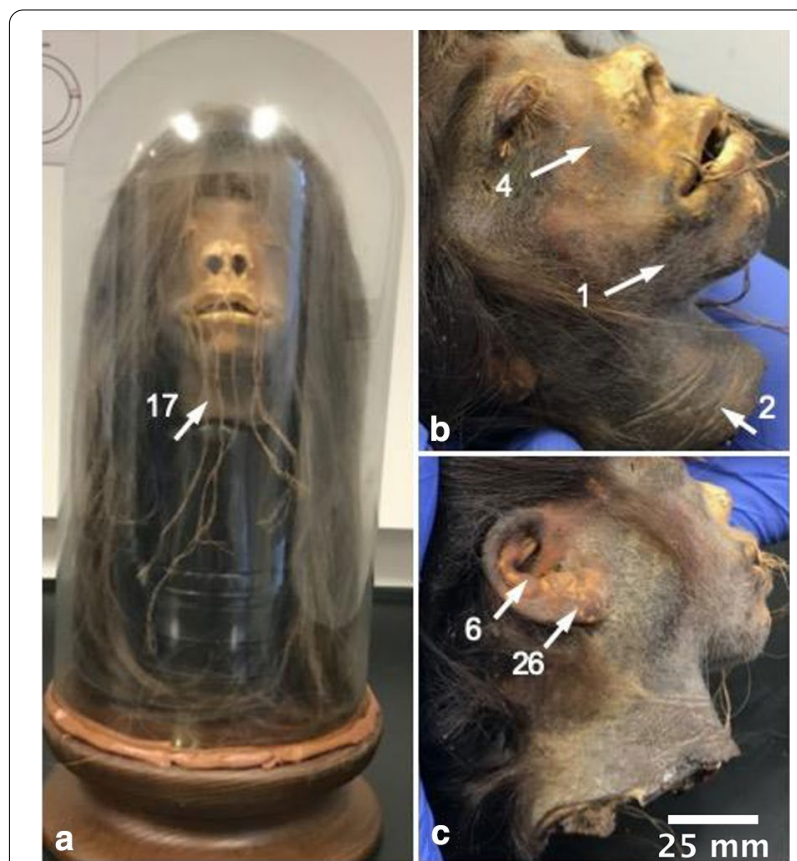

Fig. 1 Tsantsa of probable Ecuadorian origin as found in Mercer University's cultural museum collection in 2018. a The tsantsa was preserved resting under a plastic bell jar secured to a wooden base with adhesive. $\mathbf{b}$ Skin characteristics of the tsantsa: darkened skin color, leathery skin texture at neck opening, and smooth cheeks. c Anatomy of the tsantsa ears were well preserved and no evidence of ear piercing. Numbers correspond to Tsantsa Authentication Checklist in Table 1.

black powder may be an indication of remaining ash or charcoal, it also may have originated from degradation of the newspaper and/or ink.

\section{Comparison of the Mercer Tsantsa to Ceremonial Tsantsa characteristics}

Table 1 provides the checklist of criteria applied to the tsantsa, generated from the peer-reviewed literature and requirements from the INPC. This investigation was not designed to be scholarly in nature; the purpose was to repatriate the tsantsa to Ecuador and documenting the characteristics for authentication provided by the INPC was essential. The table is organized into the following categories: skin; structure and facial anatomy; modifications, and hair.

\section{Analysis of the skin}

Because ash was rubbed into the skin during the final stages of curing, the outer surface has a mahogany-black color (Fig. 1b). The skin was substantially thick and leathery, particularly noticeable at the neck, with no facial painting observed (Fig. 1b). The nose tip and cheeks were 
relatively smooth and lighter in color than the rest of the face, and downy hair was absent on the cheeks (Fig. 1b). The lack of hair on the cheeks is known to result from the use of hot rocks to "iron" the facial skin. The lighter shaded areas are consistent with a polished appearance accompanied by the breakdown of the outer epidermal layer. The combination of ironing and the smoking of tsantsas over open fires to discourage and prevent insect activity may explain the darkening of the skin $[8,12]$.

\section{Analysis of the structure and facial anatomy}

Assessment of the facial anatomy of the Mercer tsantsa was conducted during the initial inspection as well as through the use of computerized tomography (X-Ray CT). The tsantsa had the structural hallmarks of a ceremonial artifact. After processing, the size of the head was appropriate for a ceremonial tsantsa, being "no larger than a clenched adult human fist." The neck's opening had an oval shape, though somewhat distorted from the adhesive used to fix the tsantsa to the Styrofoam base. The tissue of the ears and nose were appropriately shaped presumably due to the presence of elastic and hyaline cartilages in these respective locations. Although smaller in size (like the head) the details of the ear were maintained (Fig. 1b, c). Both eyes were tightly closed, and no stitches, pitch, or adhesive was noted on the eyelids. The upper eyelid appears to be positioned over the lower eyelid. If the eyelids were indeed stitched shut then it might have been because of fine stitching known to be associated with commercial production. If this were determined to be true, it would not be consistent with authentic ceremonial production methods.

The nose itself was upturned and worn, indicative of handling as seen with traditional processing of ceremonial heads (Fig. 2a, c). Similarly, indents at the temples and protruded lips were observed and are evidence of handling during fabrication. However, the moderate degree of lip protrusion is not as exaggerated as in the fashion sometimes performed by SAAWC culture group members. The overall shape of the head is consistent with the description of being laterally pinched at the temples (Fig. 2d). These characteristics, minus the protruded lips, contribute to the Mercer tsantsa's narrow head and elongated profile (Fig. 2c), typical of ceremonial tsantsas.

CT scanning allowed for viewing intricate details of the structure of the head not discernable by simple inspection, and has been previously used to identify characteristics of ceremonial tsantsas [2, 12]. The CT scan of the tsantsa had a voxel resolution of $115 \mu \mathrm{m}$. At this resolution, the majority of the anatomical features of the tsantsa were detected; however, very fine filamentous features such as some facial hairs and eyelashes were at the limits of detection. Micro-CT scanners, can yield voxel resolution less than $100 \mu \mathrm{m}$, but we did not have access to such an instrument.

The scan revealed that the head was self-supporting, and no wires or synthetic supports were used during construction of the tsantsa. Similarly, no bones or teeth were present in the head. CT-scans also allow for examination of the layers in the skin under the epidermis, if present. Houlton and Wilkinson [2] identify that the papillary layer often detaches from the reticular layer of the dermis during traditional processing. This "double hiding" is common in the leather industry, and was present in all ceremonial tsantsas analyzed in their study. The Mercer tsantsa does not have evidence of double hiding. Image processing revealed a single layer of integumentary tissue, presumably the papillary layer of the dermis, which is visible in the $3 \mathrm{D}$ volume rendering of a sagittal section (Fig. 3i) and in 2D CT scan images (Fig. 3f).

Of note, the head possesses a unique anatomical feature: a distinct discrepancy between the height of head surface on the left of the posterior stitching line and the height of the surface on the right of the line (Fig. 3g). Due to this tissue height difference, when the 2D sagittal views of the CT scan are generated at low resolution they have a similar appearance to double hiding (Fig. 3a-c). This initially misled investigators here, but what appeared to be double hiding is not observed when the $2 \mathrm{D}$ sagittal views of this unique anatomy are generated at peak resolution (i.e. $625 \mu \mathrm{m}$ slices). Care must be taken to select appropriate data processing parameters to yield as precise image outputs as possible, otherwise unique anatomical features of a tsantsa may be incorrectly identified as double hiding as a result of image processing.

\section{Analysis of traditional fabrication and modification}

Modifications of the skin and anatomy of the tsantsa are incurred both during the life of the victim and the processing of the tsantsa. The most obvious modifications to the Mercer tsantsa are in line with those found in ceremonial tsantsas. Three holes in the upper and lower lips, joined together using a vegetal fiber, are also present in ceremonial examples (Fig. 1a). The mouth was slightly opened (Fig. 2c). During examination it became clear that the vegetal fibers strung through the lips had been damaged and degraded over time. Sections of the fibers that broke off during inspection were collected and stored separately from the head. It is difficult to determine if the mouth had been bound into a tighter closure when it was originally collected, as to be expected in an authentic artifact. Larger chonta pins (wooden thorns) were not observed and there were not significantly large perforations or distortions around the holes. Neither were there lengthy tassels made of more substantial vegetal fibers. However, it is possible that these indicators do not 

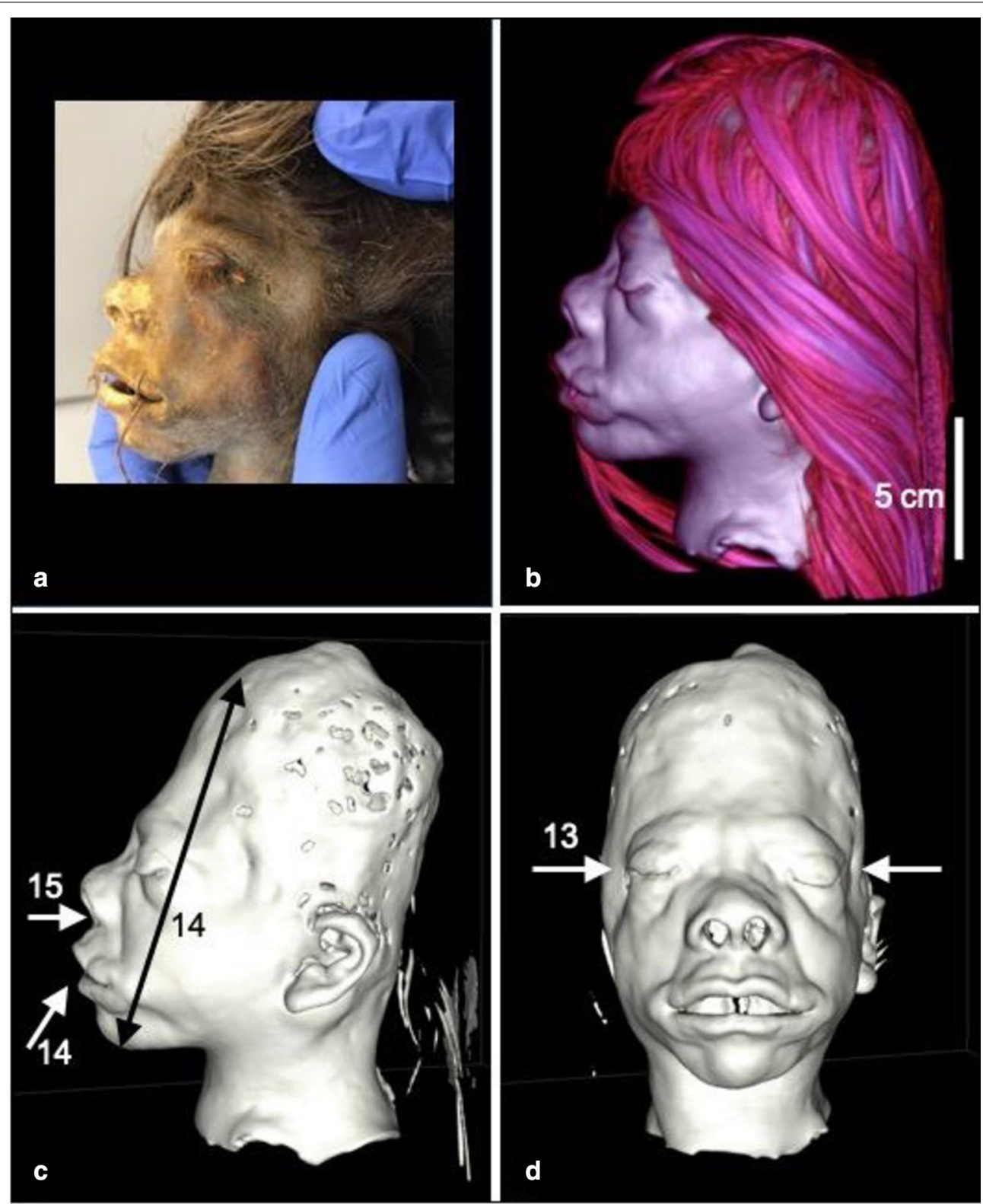

Fig. 2 Key anatomical features of tsantsa of probable Ecuadorian origin. a Profile of tsantsa with hair lifted off side of face. b 3D volume rendering of tsantsa CT scan. $\mathbf{C}$ and $\mathbf{D}$ Surface renderings of tsantsa CT scan. Tsantsa is $\sim 12 \mathrm{~cm}$ tall i.e. the size of an adult fist. Virtual removal of the tsantsa's hair revealed an elongated profile. Visible artifacts of handling during preparation and preservation include extension of the mouth, distended lips and superiorly turned nostrils. Also evident are distinct depressions at the temples

reflect the original state of the collected tsantsa. There are no photographs of it prior to it being used in filming a motion picture.

Although distorted by the adhesive used to fix the head to the base for display, the head was clearly separated from the body at or near the base of the neck at the level of the clavicles. The margin of the neck had no fibers sewn into it. While there was evidence of sawing on the internal surfaces at the base of the neck, the edge was relatively smooth and comparable to photos of ceremonial tsantsas in the literature [2]. We cannot eliminate the possibility that any fibers sewn into the neck's edge may have been simply cut off at some point after the tsantsa was preserved, or perhaps damaged when attached to the mount for display. As noted, this tsantsa was used as a prop for a motion picture. During 

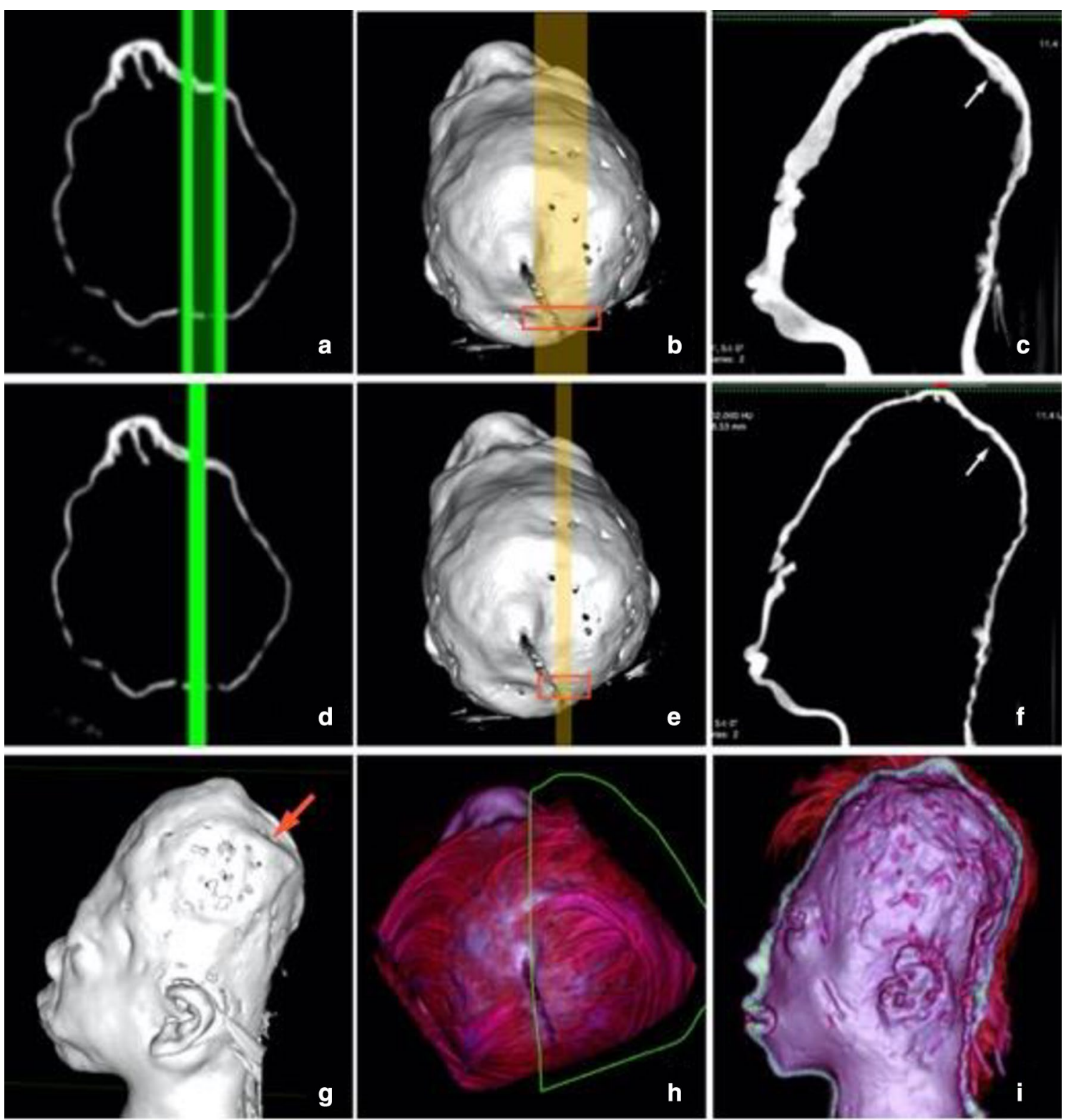

Fig. 3 Processing and interpretation of CT results. a-c Coronal plane views and sagittal plane rendering generated with Osirix MIP Thick Slab mode set to $10 \mathrm{~mm}$ sections. $\mathbf{d}$-f Coronal plane views and sagittal plane rendering generated with Osirix MIP Thick Slab mode set to $2 \mathrm{~mm}$ sections. $\mathbf{g}$ Unique depression in tsantsa head (red arrow). Height discrepancy between depression on left side of suture line and head surface on right side of suture line results in the double hiding-like appearance when the CT data is processed in $>7 \mathrm{~mm}$ sections. $\mathbf{h}$ Location of sagittal cut in 3D volume rendering for visualization of double hiding. $\mathbf{i} 3 \mathrm{D}$ volume rendering after sagittal cut shows only papillary layer remaining in tsantsa head

filming, the head was clearly attached to a small body. We can only assume that certain authentic tsantsa characteristics might have been damaged as a result of this type of use.

Internally, the observable dermis around the neck opening showed evidence of scraping. Stitches of vegetal fiber were visible down the posterior vertical midline of the head and neck (Fig. 4c, d). The stitching pattern was an uneven "baseball stitch" (Fig. 4d), so named for its similarity to the figure- 8 stitching on a baseball [2]. Again, the images derived from the CT-scan proved invaluable to the analysis. The length and density of the hair on the Mercer tsantsa complicated visualizing the stitches. Preliminary examination indicated that stitching at the base of the neck was looped through the skin in an "over and over" pattern. However, the CT scan clearly 

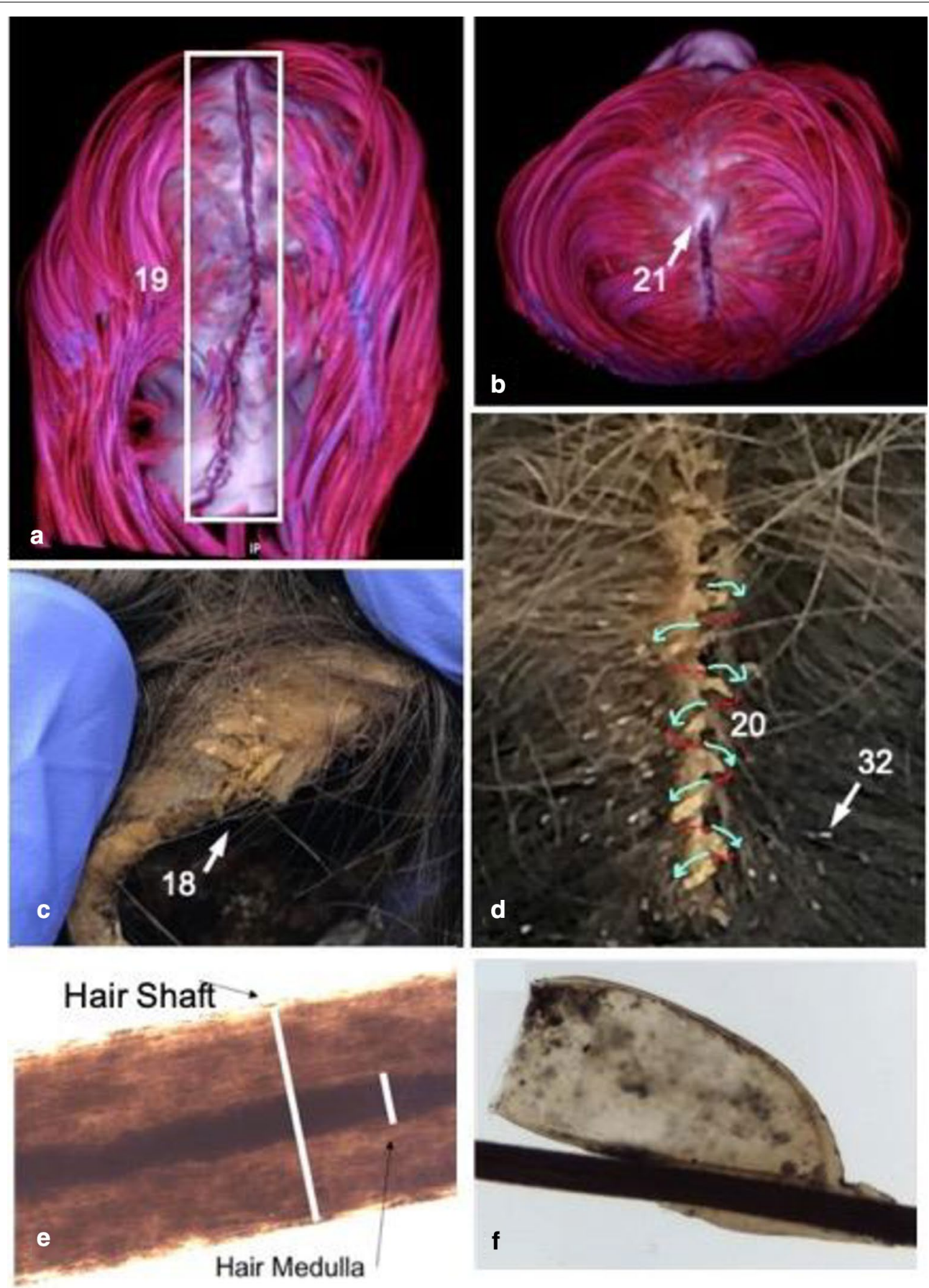

Fig. 4 Fabrication evidence and hair characterization. a 3D volume rendering showing an uneven seam of stitching down back of head. b Hole in top of head and tissue deformation indicative of hanging $\mathbf{c}$ Natural fiber sutures at neck closure $\mathbf{d}$ Sutures along back of scalp and nits in hair. Curved arrows and dashes follow the baseball stitch pattern. e Tsantsa head hair. Hair shaft width is compared to hair medulla width $(98.4 \mu \mathrm{m} \pm 18.1$ and $23.3 \mu \mathrm{m} \pm 8$, respectively) yields a hair width ratio of $0.23 \pm 0.06$. $\mathbf{f}$ An egg casing left from lice

indicates a "baseball" stitch pattern beneath the hair, not found in any of the ceremonial tsantsas described in the Houlton and Wilkinson study [2]. This stitch pattern was confirmed by careful separation of the hair on the back of the skull to examine the seam. 
The surface rendering of the CT scan data also indicated a hole at the top of the head, hidden by the hair of the subject (Figs. 2c, $d$ and $3 b$ ). This feature is observed in ceremonial tsantsas and it typically corresponds to where a string was attached. No vegetal fibers or string were observed in our tsantsa associated with this hole but these vegetal fibers, when present, are fragile and easily damaged/removed. Charlier and coworkers note that traditional preparation of a tsantsa is known to leave small amounts of charcoal or sand in the cavity, which may be visible in the CT scan [12]. No such debris was noted in the scan of the Mercer tsantsa, although a charcoal like residue was found in the newspaper that was removed from the cavity (Additional file 2: Figure S2).

\section{Analysis of the hair}

Hair is a useful factor for distinguishing between ceremonial and commercial tsantsas, and particularly identifying commercial tsantsas constructed of non-human materials [15]. The Mercer tsantsa possessed the distinctive, 3-tiered hairstyle that is consistent with hair worn by SAAWC culture group members. Small amounts of hair were present on the upper lip, between eyebrows, and within the nose. The fact that small amounts of facial hair were present in some locations (around the lips) and not in others (cheeks) is consistent with manufacturing steps that involve hair removal with hot stones during the preservation process [9-11]. Hair might remain in hardto-access places around the lips where there are folds, or where large chonta pins might have been positioned around the mouth.

Microscopic examination of hair shafts enabled accurate measurement of cross-sectional diameter of both the hair shaft and the inner hair medulla (Fig. 4e). A sample of hair shafts and their inner medulla demonstrate that the average ratio of medulla width to hair shaft width is $0.23 \pm 0.06$ (medulla width $23.3 \mu \mathrm{m} \pm 8$; hair width 98.4 $\mu \mathrm{m} \pm 18.1$ ). These samples demonstrate an average hair medulla/hair width ratio less than 0.33 , a known forensic characteristic of human hair [27]. Lice nits were also observed (Fig. 4f), providing supporting evidence that the hair is human [28].

\section{D printed replica from $\mathrm{CT}$ scan}

A to-scale 3D replica of the tsantsa was printed using a SLA printer, as they are capable of $25 \mu \mathrm{m}-100 \mu \mathrm{m}$ print resolution (per the resin choice), and they are the preferred printing modality for anatomical reproductions [29]. The 3D rendering of the DICOM file was used to generate a printable .stl of the tsantsa. SLA printing of filamentous structures longer than a few millimeters and less than $100 \mu \mathrm{m}$ in diameter is difficult, and if desired, requires extensive support scaffolds. The tsantsa's hairs, particularly its scalp hairs, posed a significant challenge for printing. Because SLA printers produce objects with continuous smooth surfaces, conversion from a 3D surface rendering into a stl file requires a program that identifies where all the 3D features connect. In the case of scalp hairs there are literally thousands of instances where the features do not connect, so the program attempts to fill in the missing connections. This connected scalp hair prevented any visualization of the features or surface of the scalp so we applied a filter to remove the scalp hair prior to generating the stl file. The .stl file was then printed in Formlabs white resin using an SLA printer (Additional file 1: File S1).

Image processing of the $3 \mathrm{D}$ rendering to remove the tsantsa's hair also removed its vegetal fibers. Thus, the 3D printed model also lacked hair and fiber stitching. The proteins found in vegetal fibers and human hair have similar structures i.e. they are predominantly comprised of alpha helical molecular structures [30,31]. This could result in similar signals on CT scans and make isolating one filamentous signal (hair) from the other (vegetal fiber) difficult during digital filtering. In some locations, filtering to fully remove the hair resulted in holes in the scalp. These instances were repaired in Meshmixer; however, they were easily identified on the scalp of the 3D printed model. Certain prominent structural characteristics were observable in the 3D printed model such as the temple indentations formed during the traditional construction of the tsantsa (Additional file 1: Figure S3A and D) and the shape of the mouth. The entire surface of the model was smooth and white, i.e. no textural characteristics of the tsantsa's skin were retained in the translation from artifact to CT scan to 3D printed model. Overall, the size of the tsantsa head and the shape of its features were maintained. Thus, a 3D printed reproduction can be useful as a replacement for an authentic artifact. Prints of tsantsas could be valuable for use in cultural and historical education either in a museum or classroom setting. The use of SLA rapid prototyping could allow for the decolonization of museum collections by repatriating such artifacts [32, 33].

Although the 3D printed model was a reasonable likeness of the Mercer tsantsa, it lacked many of the features critical for accurate evaluation by experts in the field. With alternative image processing one could virtually remove the tsantsa's hair while retaining the stitches. Other features important for characterization and authentication (e.g. skin coloring and texture and presence of facial hair) are not possible in an SLA-generated model. Whole prints of the tsantsa could be carried out where aspects such as opacity, heaviness, and overall accuracy are varied to help confer the actual qualities 
of the original specimen. Studies in the literature that attempt to understand what a typical museum-going audience might prefer in a replicated artifact demonstrate clearly that "verisimilitude" (the closeness of the printed model compared to the authentic artifact) is the quality of highest preference [29, 34, 35]. In total, the CTscan and corresponding SLA print generated here allows assessment of 12 out of 33 characteristics required for authentication. The CT scan data can be easily shared with other experts for review, whereas the rapid prototype best serves as a visual teaching aid.

\section{Repatriation of the Mercer Tsantsa to Ecuador}

Most checklist indicators of authenticity (30/33) affirm that the Mercer tsantsa is ceremonial; this includes $100 \%$ of the criteria provided by the INPC and those established by Charlier and coworkers [12]. Based upon hair analysis and the presence of nits, it is concluded that the Mercer tsantsa is of human origin. The presence of nits is in and of itself not disqualifying; however, Houlton and Wilkinson comment that only minor nit infestations are present in ceremonial tsantsas (2). With only one tsantsa available, it was impossible to determine whether the Mercer tsantsa had a minor nit infestation, or something more significant. It is also important to note that the analysis of the neck was complicated by the improper mounting of the Mercer tsantsa and its potential rough handling during the production of Wise Blood.

The Mercer tsantsa did not meet 3 requirements established by Houlton and Wilkinson. Double hiding was not confirmed via CT scan, the stitching on the back of the head was not in the typical "over and over" pattern, and the eyelids were not stitched shut; each of these criteria were met by $100 \%$ of the ceremonial tsantsas in the Houlton and Wilkinson study [2]. The authors note that a few "ambiguous" tsantsas did not possess double hiding. Additionally, certain ambiguous tsantsas also possessed baseball stitching. Although the Mercer tsantsa may have been classified as an ambiguous tsantsa according to these criteria, it met the authentication criteria established by the INPC. An Ecuadorian archaeologist from the Ministry of Cultural Heritage reviewed our report and supporting images, and issued a separate document confirming the findings.

\section{Conclusion}

The tsantsa from Mercer University's institutional holdings was repatriated on June 12th, 2019 to the General Consulate of Ecuador in Atlanta, Georgia. The methodology detailed herein was successfully employed in establishing and verifying the provenance of this item and its cultural significance. The application of CT scanning technology for the non-destructive imaging of cranial integumentary tissues was critical to the accurate identification of component manufacturing features. The omnibus list of authenticating tsantsa indicators presented here can supplement the study of other such artifacts of human remains that remain in public and private collections. Additionally, the digital scan data of the Mercer tsantsa may be shared with other institutions attempting to repatriate their own such artifacts while hoping to retain a volumetric replica. For institutions whose mission is to accurately interpret cultural heritage to acknowledge their repositories of colonialism, is a significant change in the status quo. We encourage those who hold such collections to consider including copies of other cultural heritage artifacts in online data repositories containing their $3 \mathrm{D}$ digital printing files.

\section{Abbreviations}

CT: Computed tomography; DICOM: Digital Imaging and Communications in Medicine; INPC: Instituto Nacional de Patrimonio Cultural; IRB: Institutional Review Board; mAs: Milliamperes; MIP: Maximum intensity projection; SAAWC: Shuar, Achuar, Awajún/Aguaruna, Wampís/Huambisa, and Candoshi-Shampra culture groups indigenous to South America's Amazon River Basin; SLA: Stereolithography

\section{Supplementary Information}

The online version contains supplementary material available at https://doi. org/10.1186/s40494-021-00518-z.

Additional file 1: Figure S1. Interactive Stereolithography File. This file allows one to interact with a replica of the tsantsa by rotating and exploring outer and inner surfaces. Note that hair and stitching filaments have been removed.

Additional file 2: Figure S2. Newspaper, Sand, and Ash found inside Mercer tsantsa on Initial Inspection. In this photograph a Spanish language newspaper is depicted, presumably from when it was collected. Close inspection reveals a dusty, charcoal residue that collected in small quantities within the white tray (background). Figure S3. To-Scale 3D Printed Replica of a Repatriated Ecuadorian tsantsa. A) Anterior view of the $3 \mathrm{D}$ printed replica. Numbered arrows indicate indentations formed due to pressure applied by fingers during traditional construction B) Locations on the skull that required repairs in the .stl file were clearly visible on the tsantsa replica. C) Image processing to remove the tsantsa hair was necessary to generate a printable .stl file. Removing the hair also removed all stitches. D) Demonstration of finger placement at location 1, 2, and 3, that resulted in indentations in the head. Scale bar is $2.5 \mathrm{~cm}$.

\section{Acknowledgements}

The authors thank representatives from the Ecuadorian government, particularly Marco Benitez Flores of the Ecuadorian Embassy to the United States, for facilitating the repatriation of the tsantsa to Ecuador. The authors are also thankful to Alpha Bond, whose knowledge of the origins of the tsantsa and whose tireless efforts helped ensure the tsantsa get returned to Ecuador. Also, we thank Alana Alvarez for critical language translation during meetings.

\section{Authors' contributions}

CDB and AMK were responsible for the conceptualization and administration of this project. CDB, AMK, and JT developed the methodology for all experiments AMK, JT, SP, ALF, TA collected and evaluated the computerized tomography. CDB, AMK, and AJ developed the authentication checklist and compared the Mercer tsantsa against the checklist. CDB, AMK, JT, and SP 
prepared figures and tables. CDB, AMK, JT drafted the original draft of the paper, with all authors contributing to the review and editing of the final manuscript. All authors read and approved the final manuscript.

\section{Funding}

No external funding was received for this study.

\section{Availability of data and materials}

With permission from the INPC, all data are available to scholars for research and education purposes as permitted by the corresponding author. Relevant data are found within the manuscript and its Supporting Information files. All scan files are available for education and research purposes upon reasonable request via the MorphoSource database (Media: M85767; https://www.morph osource.org).

\section{Declarations}

\section{Competing interests}

The authors (C.D.B., A.M.K., J.T., S.P., A.J., T.A.) have declared that no competing interests exist. A.F. is employed by GE Healthcare. The funder provided support in the form of salaries for author A.F., but did not have any additional role in the study design, data collection and analysis, decision to publish, or preparation of the manuscript.

\section{Author details}

'Department of Biology, College of Liberal Arts and Sciences, Mercer University, Macon, GA, USA. ${ }^{2}$ Department of Chemistry, College of Liberal Arts and Sciences, Mercer University, Macon, GA, USA. ${ }^{3}$ Biomedical Engineering, School of Engineering, Mercer University, Macon, GA, USA. ${ }^{4}$ GE Healthcare, Jacksonville, FL, USA. ${ }^{5}$ Baptist MD Anderson Cancer Center, Jacksonville, FL, USA.

Received: 7 September 2020 Accepted: 14 December 2020 Published online: 11 May 2021

\section{References}

1. Descola P. In the society of nature: a native ecology in Amazonia. Cambridge: Cambridge University Press; 1996. https://www.google.com/ search?q=In+the+society+of+nature\%3A+a+native+ecology+in+ Amazonia.\&oq =In+the+society+of+nature\%3A+a+native+ecology+ in +Amazonia.\&aqs=chrome..69i57.570j0j4\&sourceid=chrome\&ie=UTF8. Accessed 28 May 2020

2. Houlton TMR, Wilkinson CM. Recently identified features that help to distinguish ceremonial tsantsa from commercial shrunken heads. J Cult Herit. 2016;20:660-70.

3. Houlton TMR, Wilkinson C. Facial preservation following extreme mummification: Shrunken heads. Forensic Sci Int. 2018;286:31-41.

4. Rubenstein SL. Shuar migrants and shrunken heads face to face in a New York museum. Anthropol Today. 2004;20:15-8.

5. Up de Graff FW. Head hunters of the Amazon; seven years of exploration and adventure [Internet]. Garden City, N.Y.: Garden City publishing co., inc.; 1929. https://catalog.hathitrust.org/Record/001465450. Accessed 28 May 2020.

6. Hendricks JW. Power and Knowledge: Discourse and Ideological Transformation among the Shuar. Am Ethnol. [Wiley, American Anthropological Association]; 1988;15:216-38.

7. Harner MJ. The Jívaro, people of the sacred waterfalls. 1st University of California Press ed. Berkeley: University of California Press; 1984.

8. Stirling MW. Head Hunters of the Amazon. Sci Mon. 1933;36:264-6.

9. Jandial R, Hughes SA, Aryan HE, Marshall LF, Levy ML. The science of shrinking human heads: tribal warfare and revenge among the South American Jivaro-Shuar. Neurosurg Oxford Acad. 2004;55:1215-21.

10. Mutter GL. Jivaro tsantsas, authentic and forged: a study of two shrunken heads in the Mütter Museum. Trans Stud Coll Physicians Phila. 1975;43:78-82.

11. Post PW, Daniels F. Histological and histochemical examination of American Indian scalps, mummies, and a shrunken head. Am J Phys Anthropol. 1969;30:269-93.
12. Charlier P, Huynh-Charlier I, Brun L, Hervé C, de la Grandmaison GL. Shrunken head (tsantsa): A complete forensic analysis procedure. Forensic Sci Int. 2012;222:399.

13. Ross JB. Effects of Contact on Revenge Hostilities Among the Achuara Jívaro. In: Ferguson RB, editor. War Cult Environ. New York: Academic Press; 1984. p. 83-109.

14. Steel D. Trade Goods and Jivaro Warfare: the Shuar 1850-1957, and the Achuar, 1940-1978. Ethnohistory New York: Duke University Press. 1999:46:745-76.

15. Sauvageau A, Kremer C, Brochu V, Julien F, Racette S. Jivaro Tsantsas or shrunken head: an expertise of authenticity evaluation. Am J Forensic Med Pathol. 2009;30:72.

16. Taylor AC. Remembering to Forget: Identity, Mourning and Memory Among the Jivaro. Man. [Wiley, Royal Anthropological Institute of Great Britain and Ireland]; 1993;28:653-78.

17. Blankenship G. Old Animal Specimens May Hold The Key To New Discoveries. NPR.org. 2018. https://www.npr.org/2018/04/13/597740866/ old-animal-specimens-may-hold-the-key-to-new-discoveries. Accessed 11 Jan 2020.

18. James Ostelle Harrison. The Other Life of a University Professor. Gray, Georgia: PBS Printing; 2000.

19. Kakaliouras AM. An anthropology of repatriation: contemporary physical anthropological and native american ontologies of practice. Curr Anthropol. 2012:53:S210-21.

20. Native American Graves Protection and Repatriation Act. 1990. https:// www.nps.gov/nagpra/mandates/25usc3001etseq.htm. Accessed 5 Feb 2019.

21. John Huston. Wise Blood. 1979. http://www.imdb.com/title/tt0080140/. Accessed 5 Feb 2019.

22. Shrunken Head Makes Movie Debut. Macon Telegr. GA: Macon; 1979.

23. Philippe C. Complete forensic procedure for shrunken head (tsantsa) authentification.2013. https://www.nature.com/protocolexchange/proto cols/2815. Accessed 5 Feb 2019.

24. Houlton TMR. A morphometric investigation into shrunken heads. J Cult Herit. 2018;32:238-47.

25. Hermon D, Gafny R, Zamir A, Hadas L, Faerman M, Bar-Gal GK. The genetic signature of a shrunken head. Archaeol Anthropol Sci. 2011;3:223.

26. Piniewska D, Sanak M, Wojtas M, Polanska N. The genetic evidence for human origin of Jivaroan shrunken heads in collections from the Polish museums. Int J Legal Med. 2017;131:643-50.

27. Robertson JR, editor. Forensic Examination of Hair [Internet]. 0 ed. CRC Press; 1999 [Accessed 2020 May 10]. https://www.taylorfrancis.com/ books/9780203483527.

28. Reichenpfader B, Buzina W, Roll P. The mystery of shrinking heads. Forensic Sci Int Suppl Ser. 2009;1:22-3.

29. Fiorenza L, Yong R, Ranjitkar S, Hughes T, Quayle M, McMenamin PG, et al. Technical note: The use of 3D printing in dental anthropology collections. Am J Phys Anthropol. 2018;167:400-6.

30. Yang F-C, Zhang Y, Rheinstädter MC. The structure of people's hair. PeerJ. PeerJ Inc.; 2014;2:e619.

31. Klemm D, Heublein B, Fink H-P, Bohn A. Cellulose: Fascinating Biopolymer and Sustainable Raw Material. Angew Chem Int Ed. 2005;44:3358-93.

32. Isaac G. Perclusive Alliances: Digital 3-D, Museums, and the Reconciling of Culturally Diverse Knowledges. Curr Anthropol. The University of Chicago Press; 2015;56:S286-96.

33. Oxford Museum Permanently Removes Controversial Display of Shrunken Heads | Smart News | Smithsonian Magazine. https://www.smithsonia nmag.com/smart-news/oxford-museum-moves-controversial-humanremains-storage-180975805/. Accessed 20 Nov 2020.

34. Wilson PF, Stott J, Warnett JM, Attridge A, Smith MP, Williams MA. Museum visitor preference for the physical properties of 3D printed replicas. J Cult Herit. 2018;32:176-85

35. Wilson PF, Griffiths S, Williams E, Smith MP, Williams MA. Designing 3-D prints for blind and partially sighted audiences in museums: exploring the needs of those living with sight loss. Visit Stud Routledge. 2020;23:120-40.

\section{Publisher's Note}

Springer Nature remains neutral with regard to jurisdictional claims in published maps and institutional affiliations. 